\title{
SMALL EIGENVALUES OF LARGE HANKEL MATRICES
}

\section{HAROLD WIDOM ${ }^{1}$ AND HERBERT WILF ${ }^{2}$}

In this note we shall determine the asymptotic behavior as $N \rightarrow \infty$ of the smallest eigenvalue of the Hankel matrix

$$
H_{N}=\left(c_{m+n}\right) \quad m, n=0, \cdots, N .
$$

It is assumed that the $c_{n}$ are the moments of a distribution function $\alpha(x)$ on the finite interval $[a, b]$,

$$
c_{n}=\int_{a}^{b} x^{n} d \alpha(x)
$$

where $w(x)=\alpha^{\prime}(x)$ satisfies

$$
\int_{a}^{b} \frac{\log w(x)}{(x-a)^{1 / 2}(b-x)^{1 / 2}} d x>-\infty .
$$

We shall see that for the smallest eigenvalue $\lambda_{N}$ of $H_{N}$ there is an asymptotic formula of the form

$$
\lambda_{N} \sim \rho N^{1 / 2} \sigma^{-2 N}
$$

where $\rho$ and $\sigma$ are constants which will be explicitly determined. In the case of the Hilbert matrix $\left(c_{m}=1 /(m+1)\right)$ a partial result was obtained by Todd in [3]. (In certain exceptional cases the exponent $\frac{1}{2}$ must be replaced by $\frac{1}{4}$.) It will be found that $\sigma$ depends only on the interval $[a, b]$.

It will be assumed throughout that $a+b \geqq 0$. This entails no loss of generality since the Hankel matrix corresponding to the distribution function $-\alpha(-x)$ on $[-b,-a]$ has exactly the same eigenvalues as $H_{N}$.

Lemma 1. Let $P_{n}(x)(n=0,1, \cdots)$ denote the orthogonal polynomials associated with $\alpha(x)$. Then $H_{N}^{-1}$ is similar to the matrix whose $m$, $n$ entry is

$$
a_{m, n}=\frac{1}{2 \pi} \int_{0}^{2 \pi} P_{m}\left(e^{i \theta}\right) P_{n}\left(e^{i \theta}\right)^{*} d \theta, \quad m, n=0, \cdots, N .
$$

Proof. Write $P_{n}(x)=\sum_{i=0}^{n} b_{n, i} x^{i}$. Then

Received by the editors June 1, 1965.

1 Supported in part by Air Force grant AFOSR 743-65.

2 Supported in part by the National Science Foundation. 


$$
\delta_{m, n}=\int_{a}^{b} P_{m}(x) P_{n}(x) d \alpha(x)=\sum_{i, j=0}^{N} b_{m, i} c_{i+j} b_{n, j}
$$

and so if $K_{N}$ denotes the matrix

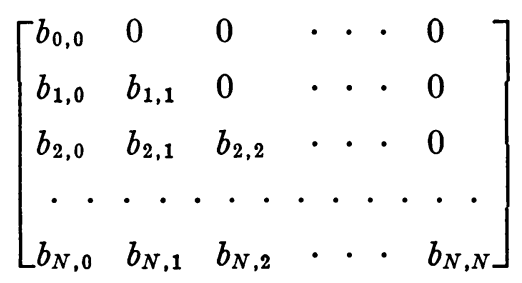

we have $I=K_{N} H_{N} K_{N}^{T}$. Thus $H_{N}^{-1}=K_{N}^{T}\left(K_{N} K_{N}^{T}\right)\left(K_{N}^{T}\right)^{-1}$. But the $m, n$ entry of $K_{N} K_{N}^{T}$ is

$$
\sum_{i=0}^{N} b_{m, i} b_{n, i}=\frac{1}{2 \pi} \int_{0}^{2 \pi} P_{m}\left(e^{i \theta}\right) P_{n}\left(e^{i \theta}\right) * d \theta
$$

which proves the lemma.

We shall be concerned now with the asymptotic behavior of $a_{m, n}$ as $m, n \rightarrow \infty$. This will turn out to be simple enough to enable us to deduce the asymptotic behavior of the largest eigenvalue of $\left(a_{m, n}\right)$.

LEMma 2. We have, uniformly for $z$ bounded away from the interval $[a, b]$,

$$
P_{n}(z) \sim(b-a)^{-1 / 2} \pi^{-1 / 2} \zeta^{n} A(\zeta),
$$

where

$$
\zeta=\frac{2}{b-a} z-\frac{b+a}{b-a}+\left[\left(\frac{2}{b-a} z-\frac{b+a}{b-a}\right)^{2}-1\right]^{1 / 2}
$$

(the square root denoting that branch which is positive for large positive z), $A(\zeta)$ is analytic in $|\zeta|>1$ and

$$
\begin{array}{r}
\log \left|A\left(\rho e^{i \phi}\right)\right|=-\frac{1}{2 \pi} \int_{-\pi}^{\pi} \log \left[w\left(\frac{b-a}{2} \cos t+\frac{b+a}{2}\right)|\sin t|\right] \\
\frac{\rho^{2}-1}{1-2 \rho \cos (\phi-t)+\rho^{2}} d t .
\end{array}
$$

Proof. If $a=-1, b=1$ this is Theorem 12.1.2 of [2] if $\alpha(x)$ is absolutely continuous and is Theorem 9.3 of [1] for general $\alpha$. The case of the interval $[a, b]$ may be reduced to this by a linear change of variable since if $q_{n}(x)$ are the orthogonal polynomials associated 
with the distribution function

$$
\alpha\left(\frac{b-a}{2} x+\frac{b+a}{2}\right)
$$

on $[-1,1]$ then

$$
P_{n}(x)=q_{n}\left(\frac{2}{b-a} x-\frac{b+a}{b-a}\right) .
$$

We omit the details.

In view of Lemma 2 we expect that the asymptotic behavior of $a_{m, n}$ depends on the maximum of $|\zeta(z)|$ as $z$ runs over the unit circle. The next lemma will describe this maximum. It is convenient at this point to distinguish three cases:

Case 1. $a>-b /(1+2 b)$.

Case 2. $a=-b /(1+2 b)$.

Case 3. $a<-b /(1+2 b)$.

Lemma 3. The maximum value of $g(\theta)=\left|\zeta\left(e^{i \theta}\right)\right|$ is given by

$$
\sigma= \begin{cases}\frac{b+a+2}{b-a}+\left[\left(\frac{b+a+2}{b-a}\right)^{2}-1\right]^{1 / 2} & \text { Cases } 1 \text { and } 2, \\ \left(\frac{1}{|a| b}+1\right)^{1 / 2}+\left(\frac{1}{|a| b}\right)^{1 / 2} & \text { Cases } 2 \text { and } 3 .\end{cases}
$$

In Cases 1 and 2 the maximum occurs at $\theta=\pi$ (and only there $\bmod 2 \pi$ ) and in Case 3 at $\theta= \pm \theta_{0}$ (and only there $\bmod 2 \pi$ ) where

$$
\cos \theta_{0}=\frac{b+a}{2 a b} .
$$

Moreover in Case 1 we have $g^{\prime \prime}(\pi) \neq 0$, in Case 2 we have $g^{\prime \prime}(\pi)=0$ but $g^{i v}(\pi) \neq 0$, and in Case 3 we have $g^{\prime \prime}\left(\theta_{0}\right) \neq 0$.

The proof of the lemma is completely elementary and need not be reproduced here.

Lemma 4. There is a constant $A$, depending only on the distribution function $\alpha(x)$, such that for all $m, n$

$$
\left|a_{m, n}\right| \leqq \begin{cases}A(m+n+1)^{-1 / 2} \sigma^{m+n} & \text { Cases } 1 \text { and } 3, \\ A(m+n+1)^{-1 / 4} \sigma^{m+n} & \text { Case } 2 .\end{cases}
$$

Proof. It follows from Lemma 2 that as long as the unit circle 
does not intersect the interval $[a, b]$ we have

$$
\left|a_{m, n}\right| \leqq \operatorname{const} \int_{0}^{2 \pi} g(\theta)^{m+n} d \theta
$$

and the desired conclusions follow readily from Lemma 3 using standard techniques.

To show that the same estimates hold even if the unit circle does intersect $[a, b]$ let us assume that 1 belongs to the interval but -1 does not. (The case in which they both belong to the interval is more complicated in only a trivial way.) We can write, for any $\epsilon>0$

$$
\left|a_{m, n}\right| \leqq \frac{1}{2 \pi} \int_{-\epsilon}^{\bullet}\left|P_{m}\left(e^{i \theta}\right) P_{n}\left(e^{i \theta}\right)\right| d \theta+\frac{1}{2 \pi} \int_{\epsilon}^{2 \pi-\epsilon}\left|P_{m}\left(e^{i \theta}\right) P_{n}\left(e^{i \theta}\right)\right| d \theta .
$$

Since the asymptotic formula of Lemma 2 holds uniformly for $\epsilon \leqq \theta \leqq 2 \pi-\epsilon$, the last integral will satisfy the estimate in the statement of the lemma. To estimate the first integral, denote by $R_{\mathrm{e}}$ the rectangle with vertices $e^{ \pm i \epsilon}, 1 \pm i$ tan $\epsilon$. This rectangle contains the arc of the unit circle given by $|\theta| \leqq \epsilon$. Since the polynomial $P_{m}(z) P_{n}(z)$ has only real zeros (Theorem 3.3.1 of [2]) its maximum absolute value on $R_{\boldsymbol{\epsilon}}$ is attained on the horizontal sides of $R_{\boldsymbol{c}}$. On these sides we may apply the asymptotic formula of Lemma 2 , and so

$$
\limsup _{m+n \rightarrow \infty} \max _{R_{\epsilon}}\left|P_{m}(z) P_{n}(z)\right|^{1 /(m+n)}=g\left(\epsilon+O\left(\epsilon^{2}\right)\right) .
$$

Therefore we have as $m+n \rightarrow \infty$

$$
\int_{-\bullet}^{\bullet}\left|P_{m}\left(e^{i \theta}\right) P_{n}\left(e^{i \theta}\right)\right| d \theta=O\left(t^{m+n}\right)
$$

for any $t>g\left(\epsilon+O\left(\epsilon^{2}\right)\right)$. A little computation shows that $g(2 \epsilon)$ $>g\left(\epsilon+O\left(\epsilon^{2}\right)\right)$ if $\epsilon$ is small enough. Thus

$$
\int_{-e}^{e}\left|P_{m}\left(e^{i \theta}\right) P_{n}\left(e^{i \theta}\right)\right| d \theta=O\left(g(2 \epsilon)^{m+n}\right) .
$$

But $\sigma>g(2 \epsilon)$, again for sufficiently small $\epsilon$ (recall that $g(\theta)$ does not attain its maximum $\sigma$ at $\theta=0$ ), and so certainly

$$
\int_{-\epsilon}^{e}\left|P_{m}\left(e^{i \theta}\right) P_{n}\left(e^{i \theta}\right)\right| d \theta=o\left((m+n)^{-1 / 2} \sigma^{m+n}\right) .
$$

This completes the proof of the lemma.

The next lemma gives the asymptotic behavior of $a_{m, n}$ as $m, n \rightarrow \infty$. First some more notation. We write 


$$
\gamma= \begin{cases}\frac{|A(\zeta(-1))|^{2} \sigma^{1 / 2}}{2^{1 / 2} \pi^{3 / 2}\left|g^{\prime \prime}(\pi)\right|^{1 / 2}(b-a)} & \text { Case 1, } \\ \frac{3^{1 / 4} \Gamma\left(\frac{1}{4}\right)|A(\zeta(-1))|^{2} \sigma^{1 / 4}}{2^{9 / 4} \pi^{2}\left|g^{i v}(\pi)\right|^{1 / 4}(b-a)} & \text { Case 2, } \\ \frac{2^{1 / 2}\left|A\left(\zeta\left(e^{i \theta}\right)\right)\right|^{2} \sigma^{1 / 2}}{\pi^{3 / 2}\left|g^{\prime \prime}\left(\theta_{0}\right)\right|^{1 / 2}(b-a)} & \text { Case 3, }\end{cases}
$$

where $|A(\zeta)|$ is given in Lemma 2 and $\theta_{0}$ in Lemma 3. We shall write, in Case 3,

$$
\operatorname{sgn} \zeta\left(e^{i \theta_{0}}\right)=e^{i \phi_{0}} .
$$

(In Cases 1 and 2, $\operatorname{sgn} \zeta(-1)=-1$.)

Lemma 5. The following hold as $m, n \rightarrow \infty$ with $m-n$ bounded:

$$
\begin{array}{lll}
a_{m, n} \sim \gamma(-1)^{m-n}(m+n)^{-1 / 2} \sigma^{m+n} & & \text { Case 1, } \\
a_{m, n} \sim \gamma(-1)^{m-n}(m+n)^{-1 / 4} \sigma^{m+n} & & \text { Case 2, } \\
a_{m, n}=\gamma \cos (m-n) \phi_{0}(m+n)^{-1 / 2} \sigma^{m+n}+o\left((m+n)^{-1 / 2} \sigma^{m+n}\right) & & \text { Case 3. }
\end{array}
$$

Proof. Suppose the unit circle does not intersect $[a, b]$. (The case in which it does can be handled just as in the proof of Lemma 4.) Then by Lemma 2,

$$
\begin{aligned}
a_{m, n}=\frac{1}{2 \pi^{2}(b-a)} \int_{0}^{2 \pi}\left\{g(\theta)^{m+n}\left[\operatorname{sgn} \zeta\left(e^{i \theta}\right)\right]^{m-n}\left|A\left(\zeta\left(e^{i \theta}\right)\right)\right|^{2}\right. & \\
& +o\left(g(\theta)^{m+n}\right\} d \theta .
\end{aligned}
$$

In Cases 1 and 2 the maximum of $g(\theta)$ occurs at $\theta=\pi$ (and nowhere else) and the result follows from Lemma 3 using standard techniques. In Case 3 the maximum occurs at $\pm \theta_{0}$. Since

$$
\zeta\left(e^{-i \theta_{0}}\right)=\left(\zeta\left(e^{i \theta_{0}}\right)\right)^{*}, \quad|A(\zeta)|=|A(\zeta)|
$$

the conclusion in this case also follows easily from Lemma 3.

Theorem. If $\lambda_{N}$ is the smallest eigenvalue of $H_{N}$, then as $N \rightarrow \infty$,

$$
\begin{aligned}
& \lambda_{N} \sim \gamma^{-1}\left(\sigma^{2}-1\right)(2 N)^{1 / 2} \sigma^{-2(N+1)} \\
& \text { Case 1, } \\
& \lambda_{N} \sim \gamma^{-1}\left(\sigma^{2}-1\right)(2 N)^{1 / 4} \sigma^{-2(N+1)} \\
& \lambda_{N} \sim 2 \gamma^{-1}\left[\frac{1}{\sigma^{2}-1}+\left(\frac{1}{\sigma^{4}-2 \sigma^{2} \cos 2 \phi_{0}+1}\right)^{1 / 2}\right]^{-1}(2 N)^{1 / 2} \sigma^{-2(N+1)}
\end{aligned}
$$$$
\text { Case 2, }
$$

Case 3. 
Proof. We shall consider in detail only Case 3; the others are easier. Let us write

$$
\begin{aligned}
b_{m, n} & =\cos (m-n) \phi_{0} \sigma^{m+n}, \\
c_{m, n} & =a_{m, n}-\gamma(2 N)^{-1 / 2} b_{m, n} .
\end{aligned}
$$

Fix $N_{0}$ and $\epsilon$. It follows from Lemma 5 that if $m$ and $n$ are sufficiently large, but $|m-n| \leqq N_{0}$, we shall have

$$
\left|a_{m, n}-\gamma \cos (m-n) \phi_{0}(m+n)^{-1 / 2} \sigma^{m+n}\right| \leqq \epsilon(m+n)^{-1 / 2} \sigma^{m+n} .
$$

Therefore if both $m$ and $n$ exceed $N-N_{0}$ and $N$ is sufficiently large we shall have

$$
\begin{aligned}
\left|c_{m, n}\right| & =\left|a_{m, n}-\gamma \cos (m-n) \phi_{0}(2 N)^{-1 / 2} \sigma^{m+n}\right| \\
& \leqq \epsilon(m+n)^{-1 / 2} \sigma^{m+n}+\gamma \sigma^{m+n}\left[\left(2 N-2 N_{0}\right)^{1 / 2}-(2 N)^{1 / 2}\right] \\
& \leqq \epsilon N^{-1 / 2} \sigma^{m+n} .
\end{aligned}
$$

It follows from Lemma 4 that for all $m, n$

$$
\left|c_{m, n}\right| \leqq A_{1}(m+n+1)^{-1 / 2} \sigma^{m+n}
$$

where $A_{1}$ is a constant depending only on the distribution function $\alpha(x)$. Denote by $\mu_{N}$ the eigenvalue of largest absolute value of the matrix $\left(c_{m, n}\right)(m, n=0, \cdots, N)$. Then from (2) and (3) we obtain

$$
\begin{aligned}
\stackrel{2}{\mu_{N}} & \leqq \sum_{m, n=0}^{N} c_{m, n}^{2} \leqq \epsilon N \sum_{m, n=N-N_{0}}^{N} \sigma^{2(m+n)}+2 A_{1}^{2} \sum_{m=0}^{N-N_{0}} \sum_{n=0}^{N} \frac{\sigma^{2(m+n)}}{m+n+1} \\
& \leqq \frac{\epsilon^{2} \sigma^{4(N+1)}}{\left(\sigma^{2}-1\right)^{2} N}+A_{2} \frac{\sigma^{2\left(2 N-N_{0}\right)}}{2 N-N_{0}},
\end{aligned}
$$

where $A_{2}$ is another constant. If now $N_{0}$ is taken sufficiently large in comparison to $\epsilon$, this will imply for sufficiently large $N$

$$
\left|\mu_{N}\right| \leqq \frac{2 \epsilon \sigma^{2(N+1)}}{\left(\sigma^{2}-1\right) N^{1 / 2}} .
$$

Now Lemma 1 implies that $\lambda_{N}^{-1}$ is the largest eigenvalue of $\left(a_{m, n}\right)$ $(m, n=0, \cdots, N)$. It follows therefore from (1) and (4) that if $\nu_{N}$ is the largest eigenvalue of $\left(b_{m, n}\right)(m, n=0, \cdots, N)$, we have

(5) $\gamma(2 N)^{-1 / 2} \nu_{N}-\frac{2 \epsilon \sigma^{2(N+1)}}{\left(\sigma^{2}-1\right) N^{1 / 2}} \leqq \lambda_{N}^{-1} \leqq \gamma(2 N)^{-1 / 2} \nu_{N}+\frac{2 \epsilon \sigma^{2(N+1)}}{\left(\sigma^{2}-1\right) N^{1 / 2}}$

for sufficiently large $N$. Since the eigenvectors of $\left(b_{m, n}\right)$ must be linear combinations $\alpha \cos n \phi_{0} \sigma^{n}+\beta \sin n \phi_{0} \sigma^{n}$ it is easy to see that 
$\nu_{N}$ is the largest eigenvalue of

$$
\left[\begin{array}{ll}
A & B \\
B & C
\end{array}\right]=\left[\begin{array}{ll}
\sum_{0}^{N} \cos ^{2} n \phi_{0} \sigma^{2 n} & \sum_{0}^{N} \sin n \phi_{0} \cos n \phi_{0} \sigma^{2 n} \\
\sum_{0}^{N} \sin n \phi_{0} \cos n \phi_{0} \sigma^{2 n} & \sum_{0}^{N} \sin ^{2} n \phi_{0} \sigma^{2 n}
\end{array}\right] .
$$

We find that as $N \rightarrow \infty$

$$
\begin{aligned}
& A=\frac{1}{2}\left[\frac{1}{\sigma^{2}-1}+\frac{\sigma^{2} \cos 2 N \phi_{0}-\cos 2(N+1) \phi_{0}}{\sigma^{4}-2 \sigma^{2} \cos 2 \phi_{0}+1}\right] \sigma^{2(N+1)}+O(1), \\
& C=\frac{1}{2}\left[\frac{1}{\sigma^{2}-1}-\frac{\sigma^{2} \cos 2 N \phi_{0}-\cos 2(N+1) \phi_{0}}{\sigma^{4}-2 \sigma^{2} \cos ^{2} \phi_{0}+1}\right] \sigma^{2(N+1)}+O(1), \\
& B=\frac{1}{2} \frac{\sigma^{2} \sin 2 N \phi_{0}-\sin 2(N+1) \phi_{0}}{\sigma^{4}-2 \sigma^{2} \cos ^{2} \phi_{0}+1} \sigma^{2(N+1)}+O(1),
\end{aligned}
$$

and from these there follows easily

$$
\nu_{N}=\frac{1}{2}\left[\frac{1}{\sigma^{2}-1}+\left(\frac{1}{\sigma^{4}-2 \sigma^{2} \cos 2 \phi_{0}+1}\right)^{1 / 2}\right] \sigma^{2(N+1)}+O(1) .
$$

The theorem follows from (6) and (5) if we observe that $\epsilon$ was arbitrarily small.

We regret to announce that in the case of the Hilbert matrix

$$
\left(\frac{1}{m+n+1}\right) \quad(m, n=0,1, \cdots, N)
$$

our result takes the form

$$
\lambda_{N} \sim 2^{9 / 8} \pi^{3 / 2}\left(73-48(2)^{1 / 2}\right)^{-1} N^{1 / 2}\left(3+2(2)^{1 / 2}\right)^{-2 N-3 / 4} \quad(N \rightarrow \infty) .
$$

\section{REFERENCES}

1. Ya. L. Geronimus, Polynomials orthogonal on a circle and interval, Pergamon, New York, 1960.

2. G. Szegö, Orthogonal polynomials, rev. ed., Amer. Math. Soc. Colloq. Publ., Vol. 23, Amer. Math. Soc., Providence, R. I., 1959.

3. J. Todd, Contributions to the solution of systems of linear equations and the determination of eigenvalues, Nat. Bur. Standards Appl. Math. Ser. 39 (1954), 109-116.

Cornell University AND

University of Pennsylvania 